\title{
Assessment of future Autonomous Vehicle market leadership in the US
}

\author{
José Diamantino de Almeida Dourado ${ }^{1}$, Cleveland Maximino Jones ${ }^{2, *}$, Herlander Costa Alegre da Gama Afonso ${ }^{3}$, \\ Lívia de Moraes Mariano Botelho ${ }^{4}$
}

1 CEFET - Centro Federal de Educação Tecnológica Celso Suckow da Fonseca, Unidade Nova Iguaçu; diamantinodourado@hotmail.com

2 INOG/UERJ - Instituto Nacional de Óleo e Gás; clevelandmjones@gmail.com

3 CEFET - Centro Federal de Educação Tecnológica Celso Suckow da Fonseca, Unidade Nova Iguaçu, herc_afonso@yahoo.com.br

4 CEFET - Centro Federal de Educação Tecnológica Celso Suckow da Fonseca, Unidade Nova Iguaçu, liviamoraesmb@hotmail.com

* Correspondence: clevelandmjones@gmail.com; Tel.: CMJ +55-24-98127-3942

\begin{abstract}
Scientific and technological advances in telecommunications and onboard electronics, and advances in sustainability standards, dictated major changes to various industrial sectors, including the automotive industry, where hard and soft approaches to manufacturing are vying for market dominance. This work presents a prospective analysis of the autonomous vehicle (AV) market, analyzing three of the main US AV technology firms, Tesla, Waymo and Apple. Their designs and solutions are compared, and prospective scenarios were constructed based on an analysis of their strengths, weaknesses, opportunities and threats (SWOT). The results suggest that Tesla currently exhibits the greatest market leadership in the group studied. However, it was concluded that in the medium term, Waymo would surpass Tesla and assume market leadership. In the long run, it was concluded that Apple will overcome its rivals and dominate this market.
\end{abstract}

Keywords: autonomous vehicles; SWOT analysis; 3D object detection; artificial intelligence; market dominance

\section{Introduction}

According to Litman [1], in 2040, autonomous vehicles (AV) will represent $30 \%$ of the fleet and will constitute $50 \%$ of all sales. Furthermore, AV growth will continue at nearly 40\% annual rate between 2019 and 2026 [2]. These predictions indicate that AV are indeed the future of the automotive market. The AV market has grown and attracted companies from different business segments, both from the automotive sector and specialist companies involved in software and hardware development.

Currently, there is a fierce competition underway for market dominance of the AV market, involving not only companies that are part of the traditional automotive industry, but also companies that established themselves as hardware and software companies, such as Apple, Waymo (Google/Alphabet Group) and Tesla.

The AV market is so promising in terms of business opportunities and sales revenues, that Apple considered it, in 2017, "the mother of all AI (artificial intelligence) projects" [3]. The company is thus concentrating efforts on autonomous systems. Waymo, in turn, a Google subsidiary, has developed software, hardware and automobiles with that same objective, seeking to create safer and more reliable technologies for a totally autonomous vehicle [4].

Given that scenario, the key question that needs to be answered is which solution offered by those companies, based on their competitive advantages, will eventually dominate the market. Will that solution come from an established automotive industry player 
or will it come from a technology company that focuses on hardware or software development?

This work analyzes the corporate strategies and the competition for dominance in the US AV market, seeking to identify which company is likely to achieve a dominant competitive advantage, and in what relative time scale. That analysis is important, since the race for AV dominance has already started, when, in 2016, Tesla announced that its models would be self-driving [5].

In this study, a qualitative SWOT (strengths, weaknesses, opportunities, and threats) analysis was performed, since it has been successfully utilized in various different business segments [6], as well as in the AV market [7,8,9].

\subsection{Literature Review}

From ancient times, man learned to harness the power of other animals for transportation, then the power of the steam engine, and later, of the internal combustion engine [10]. The fourth industrial revolution, or Industry 4.0, began in the 21st Century, involving autonomous processes and other technologies that provided significant performance gains [11]. A new stage in transport systems was thus inaugurated, based on transport design and internet services, such as Uber and others. Advances in big data storage and handling, as well as advances in AI, opened new frontiers in business and market dominance for companies such as Tesla, Google and Apple.

\subsection{Self-driving vehicles}

A self-driving vehicle, or $\mathrm{AV}$, is a vehicle that can make driving decisions without human intervention, while offering transport for people or cargo on roads [12]. To reach that objective, AV navigation systems involve several automated steps to obtain data from the environment, identify the position of objects and avoid collisions. One of the primary requirements is the ability to sense, process and respond to dynamic and static events with appropriate speed, with performance equivalent or superior to that of human drivers. Thus, an AV needs an intelligent onboard technology, which can store, process and manage all information gathered by sensors, besides using data pre-registered in its data bank, whether local or remote.

\subsubsection{Basic operation of an AV}

The components of an AV include sensors, radars, cameras, electronic stability controllers, among others. The structure of an AV varies among carmakers and models, however they all possess a control unit that functions as a brain. The control unit is a mechatronic system that includes all onboard electromechanical controllers (injection, brake, angular position systems, etc.), and driver support systems based on applied computational solutions.

The sensor system is composed of three groups: route reconnaissance systems, object and obstacle reconnaissance systems (laser scanners, radars, ultrasonic sensors, etc.), and navigation systems (compass, gyroscope, accelerometer, speed sensor and GPS). While the object reconnaissance sensors identify obstacles to allow the vehicle to avoid collisions, the reconnaissance and navigation systems ensure that the mechatronic systems work correctly, allowing the vehicle to travel in a controlled fashion, maintaining dynamic stability and the desired course.

Actuators are responsible for controlling direction, speed, turning and other parameters. The actuators are, in turn, supported by AI softwares that have been recently developed, utilizing neural networks to create their own operational logic. These softwares can automatically learn their own logic, based on a large quantity of data, with minimal human input [13].

The sensors collect data that are processed, generating knowledge about the environment in which the vehicle is traveling. Given the knowledge about the environment, the software then creates alternatives, using AI, and makes decisions. 
There are various levels of autonomous driving (Figure 1) [14]. Currently, AV are at the level of conditional automation, where the hardware and software system drive the vehicle and monitor the environment in which it is traveling but, require engaging a human driver to assume control in risk conditions. The human driver must still drive, although a lower attention level is required. In 2020, a high automation level was expected to be available, which would monitor the environment without human intervention. However, that system would still be limited to certain conditions and environments, with weather, for example, being a limiting variable. The final stage is defined as a system with complete automation, where the system is able to handle all driving needs, on a par with human drivers.

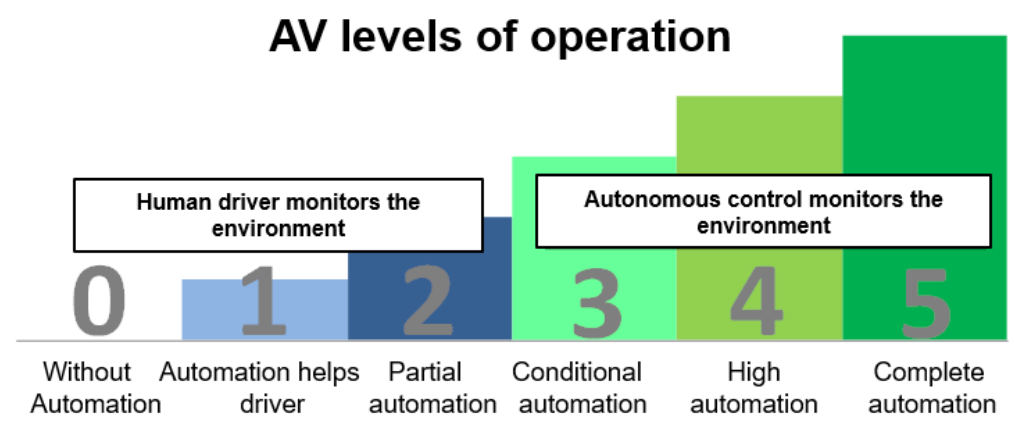

Figure 1. Levels of autonomous driving. Based on [14].

\subsubsection{Benefits and public perception of AV}

Significant benefits for society could accrue from AV technology, including convenience, cost reduction, time savings, productivity increases, accident reduction, and environmental benefits, related to reduced human driving and reduction in the number of vehicles and infrastructure needed to meet travel needs [15].

Regarding the legislative framework governing AV, different countries exhibit varying degrees of legislative readiness regarding AV. Pertinent legislation involves not only requirements for $\mathrm{AV}$ operation and driver behavior and responsibility, but also civil and criminal responsibilities in case of accidents. The relative readiness of countries regarding AV was tracked, assessing policy, legislation, technology, innovation, infrastructure, and consumer acceptance [16]. The most favorable rankings observed were among countries that have a proactive government regulatory framework, good road and mobile infrastructure, a strong private sector, a strong automotive industry, and a business environment that promotes partnerships.

Villasenor [17] argues that the judicial system should not be overburdened with additional legislation regarding AV, since existing legislation in the USA, for example, is already capable of handling legal needs involving products, manufacturers and users.

Widespread implementation and expansion of new urban mobility technology will depend on the ability of current and new legislation and specific regulatory norms to provide adequate guidance and predictability.

\section{Materials and Methods}

The present work provides information regarding AV, obtained by a thorough literature review, and an analysis of case studies of three competing companies with high technology solutions in the AV market: Tesla, Apple and Waymo. Market characteristics, current market presence, market reach, marketing strategy, financial health and technological advantages regarding manufacturing and system design were considered.

An assessment of the relative weight of these factors in influencing market dominance was also considered, to suggest ultimate market dominance.

\subsection{SWOT matrix}


To assess the relative competitiveness among companies, a SWOT analysis was performed to evaluate Strengths, Weaknesses, Opportunities and Threats. A SWOT analysis is a support tool for the decision process, and aids in the strategic planning process of companies. It considers the relation between internal (strong and weak points, controllable by the company) and external (opportunities and threats, not controllable by the company) factors that affect the company and the market in which it operates [6]. External factors that affect the AV market, were considered as of 2019, and new AV market entrants from Europe and Asia were not analyzed.

Typical external factors not controlled by companies include political and legal environment, macroeconomic situation, and technical, social and demographic drivers, which affect the opportunities and threats they face. Typical internal factors that companies can control include physical, organizational, financial and human resources at their disposal, which affect their strengths and weaknesses [6].

Given those concepts, a SWOT matrix was generated for each of the three companies studied, Tesla, Waymo and Apple.

\subsection{Data collection}

Despite limitations regarding confidentiality of information available, a SWOT matrix was constructed with qualitative data collected regarding competitive advantages of each company. All data are from secondary sources, collected from articles in periodicals, news media and corporate and institutional sites.

While mobility solutions necessarily entail mass transport solutions, AV developments generally involve individual transportation solutions. This research focused on AV for individual transportation.

\subsection{Assessment of market dominance factors}

The relative importance of factors that will ultimately influence market dominance in the short, medium and long-term was considered, and each company was assessed regarding these factors, to suggest the likelihood of ultimate market dominance for each.

\section{The companies studied}

The three companies studied were Tesla, Waymo and Apple.

\subsection{Tesla, Inc.}

Tesla was founded in 2003 by engineers that sought to prove that electric vehicles (EV) could be better than fossil fuel powered cars. Since its founding, Tesla has focused on sustainability, seeking a zero emissions future for its cars, and investing in clean energy [18].

\subsubsection{SWOT analysis - Tesla}

\subsubsection{Strong points for Tesla}

Tesla is already the current market leader in AV (autopilot vehicle) sales. Tesla's Model 3, available since 2016, has eight cameras, twelve ultrasonic sensors and a forwardlooking radar. The cameras allow $360^{\circ}$ visibility around the car, at up to 250 meters distance from it.

Tesla leads in miles of AV data collected. Tesla has collected and sought to process the data acquired by its cars (a type of crowdsourcing), regarding their real-world performance and driver reactions. It has hundreds of thousands of clients using its semi-autonomous (autopilot) technology every day, in different driving settings around the world. In 2016, Tesla registered nearly 780 million miles driven, 100 million of which were collected while the autopilot was at least partially in control [19]. Tesla collects data through its autopilot and its "shadow" mode, as well directly from its clients, allowing it to build a big data capability, with the expectation that it would add value to the development and 
improvement of their product. In "shadow" mode (when the vehicle is not performing any automatic action), the system records how the computer would act if it were in control, including how it would have acted to avoid an accident, if one occurred, thus generating data under different driving scenarios, and allowing it to compare human versus system decisions. Big data acquisition can also demonstrate the safety of the autonomous driving mode to users and regulatory agencies, through statistics gathered over thousands of miles [20].

Tesla AV sensors allow the detection of soft and hard objects at 500 meters, double the reach of the cameras. Finally, the radar receives additional data at wavelengths able to see through fog, rain, and other obstacles [21].

Tesla claims that all its vehicles leave the factory with the hardware necessary for autonomous driving.

Tesla has highly advanced, automated manufacturing plants.

Tesla has a strongly vertically integrated manufacturing process. Its manufacturing operations include machining, foundry, assembly, and other processes. The same is true for manufacturing of its car components. Even if some of its parts are outsourced, Tesla is responsible for fabrication of its lithium-ion batteries, electric motors, gearboxes, and other components. This characteristic allows it to have a greater control over its production process [22].

Tesla is already a vehicle manufacturer that integrates hardware and software in its models, from the design stage upwards.

The Tesla brand has an innovative image, never having produced fossil fuel vehicles, and is seen positively by clients who value environmental aspects.

\subsubsection{Weak points for Tesla}

Tesla international sales reach is limited, since most of its sales are in the USA, with a small part in China and developing countries [22].

Tesla's supply chain is limited internationally, which could also limit international sales.

Tesla's big data capability, according to Raj Rajkumar' ${ }^{1}$, may not provide the advantages over its competitors that Tesla expects, since it does not disclose what data is collected and how it is processed [23].

Tesla does not have the necessary processing speed to process data collected, since the advantage of having a big data capability is dependent on fast and precise utilization of that information. If that is true, its AI technology may not be performing as needed.

The autonomous driving resource included in all vehicles is not yet available to users due to regulatory issues and perhaps the need to perfect this technology.

Tesla is averse to employing LIDAR technology, being one of the very few in the AV Market that eschews that technology [24].

Tesla has had difficulty in mass producing its vehicles to meet market demand. Given so many delays and missed production targets, its credibility in a highly competitive market has been tarnished.

Tesla lost US $\$ 7,430$ per minute in 2018 , when its free cash flow was already negative for six consecutive semesters [25]. Despite past losses, its creditors, investors and clients still view the company favorably, possibly expecting future sustainable results [26]. More recently, Tesla posted vastly improved financial results for 2019 [27] and 2020 [28], however, its profit is still marginal.

\subsubsection{Opportunities for Tesla}

1 Co-director of General Motors-sponsored connected and autonomous driving research lab at Carnegie Mellon University. 
A common opportunity to all companies in the AV market is the Paris Agreement ${ }^{2}$ to mitigate damage to the environment by limiting greenhouse gas emissions. Thus, EV are a promising option. Together, EV and AV technologies could be even more attractive, since AV could diminish the number of cars on the road, besides reducing the time vehicles remain idle. Since Tesla produces only EV, it may be able to better exploit that market.

Since Tesla's current international exposure and supply chain are so limited, there may be synergies involved in expanding both its sales and supply chain internationally.

Because, unlike its competitors, Tesla does not have a vast product portfolio, it may be able to expand its portfolio and achieve further growth.

The positive attitude of its clients, who have even advanced payment for their purchases, to help the company, could allow it to capitalize on their goodwill.

The "shadow" mode capability of its vehicles could allow Tesla to identify shortcomings in the system and achieve significant AI learning, beyond what some of its competitors might be able to achieve.

Because Tesla is responsible for the integration of the driving systems of its cars, its big data capability may offer more AI learning opportunities than its competitors. That could include more thorough autopilot versus human driver experience and failure identification.

Given its manufacturing performance shortcomings, if Tesla can resolve its operational bottlenecks, it may significantly grow its production capacity.

Regarding battery technology, a common opportunity to all companies in the AV market lies in adopting the use of sodium batteries (or another technology), as opposed to lithium batteries, since sodium is abundant and would not represent a limiting supply factor [29].

\subsubsection{Threats for Tesla}

If Tesla's investment plans cannot be executed as planned, due to financial restrictions, it may fall significantly behind other competitors in the AV market.

In a strongly competitive market, new market entries represent an even more intense challenge.

Tesla, as other players in the AV Market, may face lack of acceptance by drivers who may not adapt well to onboard computers.

As is the case with other players in the AV market, Waymo may face battery issues relating to lithium scarcity, prices, and technology. Tesla batteries are based on lithium, a promising technology that has been the subject of much research. However, lithium prices are volatile, and lithium is relatively scarce. If the company does not find another source of raw material, it may have difficulty in maintaining production levels, especially if it tries to achieve mass production.

Despite its CEO Elon Musk classifying LIDAR sensors as a "crutch" [30], thus undesirable, the fact that Tesla does not utilize LIDAR, unlike most of its competitors, may adversely impact its image and market position, if its sensor technology cannot compete effectively with LIDAR.

2 The Paris Agreement was reached at the Paris Climate Conference (COP21) in December 2015, where 195 countries adopted a legally binding deal to avoid dangerous climate change (https://ec.europa.eu/clima/policies/international/negotiations/paris_en). 


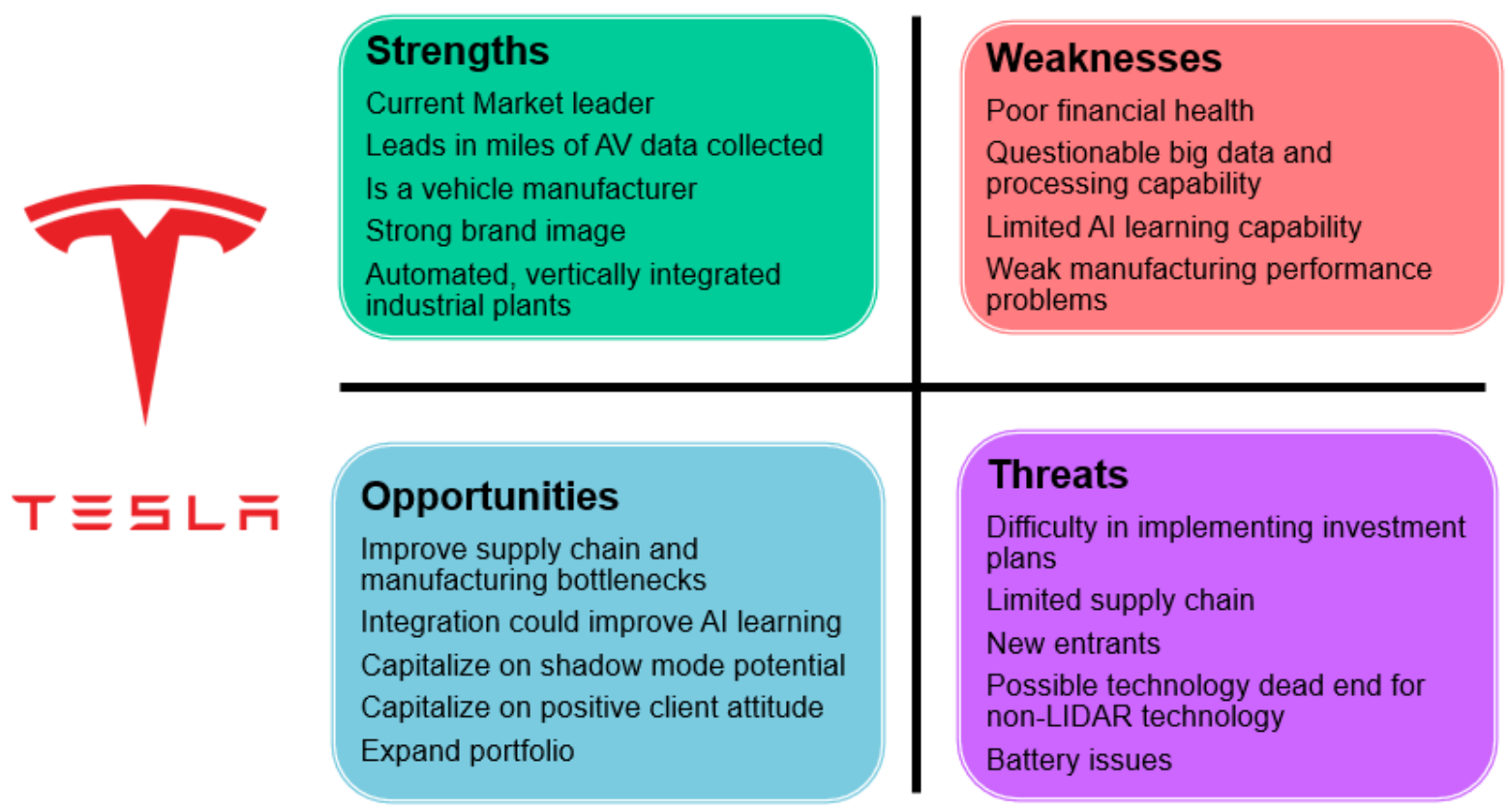

Figure 2. SWOT matrix for Tesla.

\subsection{Waymo}

Waymo was founded in 2016, with the mission of developing safe AV technology, seeking to eliminate the need for a human driver in the driver's seat. However, its work began before it was created, when Google was still researching this technology, in 2009. After Waymo became part of Alphabet, Inc., ${ }^{3}$ it has sought to make AV available to the public [4].

\subsubsection{SWOT analysis - Waymo}

\subsubsection{Strong points for Waymo}

As a brand, because it is tied to Google, Waymo has strength and credibility with investors and clients, besides having an innovative and entrepreneurial image. Furthermore, its visibility has advanced through its tests on public roads.

Waymo has experience in developing software, and can create intelligent systems for AV.

Waymo does not collect information only from the real world, but also from simulations. In early 2018, Waymo reached 5 million miles driven in public roads and 2.7 billion miles in a virtual environment, which represents a faster and more efficient means of AI learning to support its AV platform. The company utilizes its software to create and test different scenarios, collecting data for product improvement [31].

Waymo's minivans have five radar sensors, eight cameras, and three different types of LIDAR (Light Detection and Ranging) sensors. Its long-range radar sensors are located on the front and around the cars. This technology is the only one capable of seeing through fog, rain and snow, besides seeing objects. Its cameras cover all points around the vehicle, and can recognize shapes, including traffic signs. Its LIDAR sensors, positioned above the vehicle, function as laser scanners, mapping and sweeping short, medium and long distances. That technology utilizes the reflection from those lasers to detect objects and estimate distances. All those data collected allow the creation of a 3D image of the real world

\footnotetext{
${ }^{3}$ Alphabet encompasses several companies that belong or are tied to Google. Due to their growth, management decided to divide the company into smaller segments, among them Google, Waymo and others.
} 
and a $360^{\circ}$ view around the vehicle, detecting pedestrians, traffic, cyclists, and other obstacles [32].

Waymo is part of Alphabet, which includes different business segments, such as cloud computing (Google Cloud Platform), video sharing (YouTube), electronic media marketing (DoubleClick), residential automation (Nest Labs), among others, with publicity as its primary source of revenue. Thus, Waymo has strong experience in marketing software solutions.

Waymo finances are strong, since it belongs to Alphabet, a company that operates in various business segments and has a large revenue base. Alphabet's 2019 revenues were US\$162 billion, while less than 1\% were from its "other bets," including Waymo [33].

\subsubsection{Weak points for Waymo}

As a brand, Waymo is not as well-known as Google or Alphabet.

Waymo's test vehicle fleet is small, so less real-world data are accumulated. Currently, the company has only 600 minivans for testing and real-world data collection. It estimates having thousands more Chrysler Pacifica Hybrid models, after its deal with Fiat Chrysler [34].

Since Waymo is not a car manufacturer, but a software firm, as is its sister company Google, its AV fleet are sourced from third parties.

Waymo's test vehicles are hybrid cars, which are not the EV models that could better integrate with the electronics of $\mathrm{AV}$, and they do not exhibit the environmental benefits of EV.

\subsubsection{Opportunities for Waymo}

According to Reuters, on December 5th, 2018, Waymo launched an autonomous taxi service in Arizona [35]. This could represent an opportunity for Waymo to enhance its position in the AV market, in relation to its competitors.

Waymo could utilize the tools that Google Maps has developed, regarding localization, street and road data, and other real-world information, to strengthen its AV position, possibly even supplying software solutions to competitors.

If Waymo were to decide to build its own vehicles, it could overcome the disadvantage of having to source vehicles from third parties.

Waymo's strong financial position may allow it to finance new investments in $\mathrm{AV}$, to improve its competitive position.

A common opportunity to all companies in the AV market is that the Paris Agreement could stimulate AV sales.

Another common opportunity to all companies in the AV market lies in adopting the use of sodium batteries (or another technology), as opposed to lithium batteries, since sodium is abundant and would not represent a limiting supply factor [29].

\subsubsection{Threats for Waymo}

Waymo may face a threat to its entry in the AV market, since, unlike Tesla, for example, it does not have a tradition in the car market.

Waymo utilizes LIDAR laser scanning technology in its test vehicles, which is used by most competitors, thus it does not have an easy means of differentiating its product.

Waymo has limited in access to real-world information. The difference in number of test vehicles is especially glaring when Waymo's 600 test cars are compared with Tesla's 300 thousand client cars that act as test vehicles. Waymo's test vehicles only collect data in areas where AV are allowed, seriously limiting the scope of its data acquisition.

Like other players in the AV Market, Waymo may face lack of acceptance by drivers who may not adapt well to onboard computers. 
As is the case with other players in the AV market, Waymo may face battery issues relating to lithium scarcity, prices, and technology.

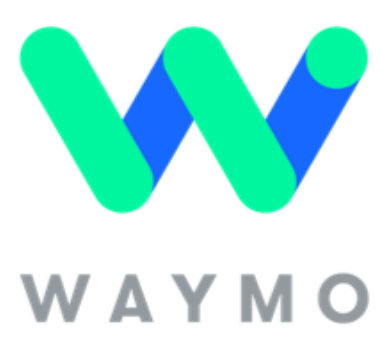

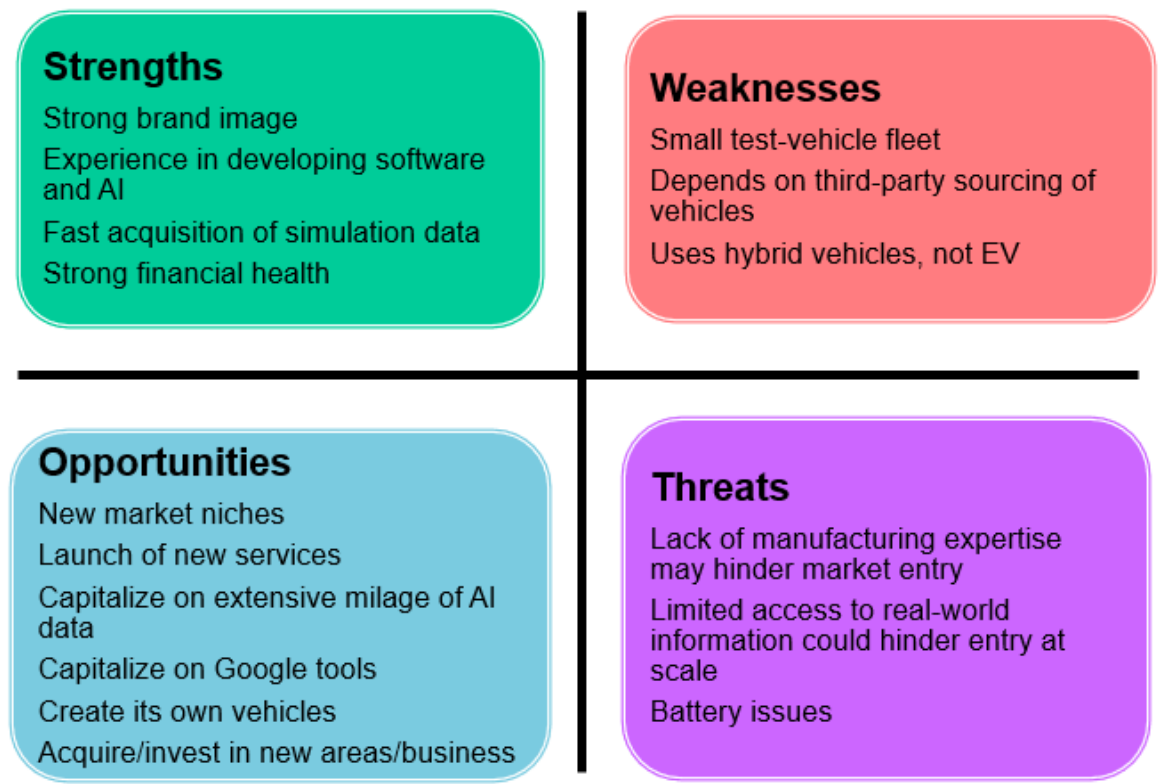

Figure 3. SWOT matrix for Waymo.

\subsection{Apple, Inc.}

Apple Inc., initially called Apple Computer, Inc., was founded in 1976 [36]. The company is known for its innovative focus, and supplies complete, integrated hardware and software solutions, computers, mobile phones and other devices. It has distinguished itself by designing its own products, such as chips, drives and monitors, and by integrating its components [37]. In 2018, Apple launched the iCar project, with codename Titan. Apple limits disclosure regarding the project, maintaining, as is typical of its product launches, strict secrecy prior to launch day.

\subsubsection{SWOT analysis - Apple}

\subsubsection{Strong points for Apple}

Apple is a very strong brand name that continues to enjoy client loyalty. Its image is strongly tied to innovation, quality, independent development of its products, and what the brand symbolizes [38]. Another characteristic that captivates its clients is that Apple ensures their absolute privacy and security [39].

Any product launched by Apple enjoys a strong advantage in relation to competing products, even in competitive markets, in which Apple is accustomed to operate.

Apple has one of the strongest financial conditions among all companies, with US\$65 billion in revenue in the quarter ending September 26, 2020 [40], while it became the first trillion-dollar company (in market valuation) in the world, in 2018 [41].

Despite almost all the design and development of its products being done in the US, Apple has a strong international presence, and almost $60 \%$ of its revenues are generated from international sales [40].

Apple made revealing choices regarding its plans to dominate the AV market. In 2017, CEO Tim Cook said that AV technology is the "mother of all AI projects" [3], and that this technology is one of the most difficult with which to work, in AI. Since 2015, Apple has been hiring AV talent, such as Doug Betts, with experience at Fiat Chrysler Automobiles, Nissan and Toyota [42], Paul Furgale, a renowned AV researcher and deputy director of the Autonomous Systems Lab at the Swiss Federal Institute of Technology (ETH), who led projects to develop AV self-parking technologies [43], and even executives from Tesla [44]. 
Apple has a strong record of software development, and has been registering patents (US2013/0099892A1, US2013/0197674A1 and others) related to AV technologies, such as for vehicular control through Apple phones. They allow the automatic regulation of seats, mirrors, air conditioning and other equipment, and include bluetooth sensors for communication between vehicles and for GPS updating of blind spots and emergency vehicles nearby [45]. These technologies facilitate the change to AV operation.

Apple publishes AV research by its employees, such as that which proposes a novel software, called VoxelNet, which helps computers to detect objects in 3D, including pedestrians and cyclists [46]. Normally, AV use a combination of 2D cameras and LIDAR to identify and measure the distance to objects, however, that method can still result in imprecise results. VoxelNet can achieve better detection by itself, and with less sensors. While LIDAR image-based detection can furnish information regarding depth, thus helping in the localization and characterization of object shapes, most existing 3D LIDARbased detection methods are based on manually created representations of objects. VoxelNet, on the other hand, claims to automatically create its own 3D image patterns through a learning process involving neural networks and convolution, and in a shorter time and with less memory, despite access to the same data as other LIDAR technologies ${ }^{4}$.

Apple's extensive experience in in-house development of its products and services, such as in the mobile telephone segment, could be valuable in integrating hardware and software for the AV market. In 2009, Apple's market share of global smartphone sales was only $16.1 \%$. However, even in the face of strong competition from new vendors, it surpassed Samsung (18.8\% share), and eventually reached a 20\% share in 2019, maintaining global leadership since then [47]. Throughout that period, Nokia, the main European company, saw its share fall, while Asian firms, such as Samsung, Huawei and Xiaomi saw their market participation rise strongly. Apple's proven strategy allowed it to remain a strong player, and even consolidate its brand in the global smartphone market.

\subsubsection{Weak points for Apple}

Apple has a small test fleet collecting real-world data, thus it is restricted to relatively less diversified environments. While the exact number of its test vehicles is unknown, it is likely smaller than that for Tesla and Waymo, despite entering a deal with Volkswagen to transform some T6 Transporter vans into AV buses, for shuttling employees between company headquarter locations [48]. Apple has only 45 autonomous test vehicles in California (Lexus RX450 hybrid SUVs), each one equipped with large LIDAR sensor racks, besides strategically positioned cameras [49].

Despite the characteristic of not outsourcing its technology, Apple has utilized vehicles made by other manufacturers, thus making it dependent on them in order to acquire real-world data.

As is the case for Waymo, the test vehicles utilized by Apple are hybrid, which are not the EV models that could best integrate with the electronics of AV, and they do not exhibit the environmental benefits of EV.

\subsubsection{Opportunities for Apple}

Apple's remarkable client loyalty and positive image could be especially important when it finally launches a consumer-ready AV product.

Apple's financial strength could also become an important competitive advantage, as it eventually enters the phase of building and marketing its own mass-produced AV product, since it could build or buy any manufacturing facilities that it needs, or enable

4 VoxelNet outperforms advanced LIDAR-based 3D detection methods by employing faster and more efficient algorithms. The inference time of the software is $225 \mathrm{~ms}$ : voxel input computation time is $5 \mathrm{~ms}$, network learning time is $20 \mathrm{~ms}$, intermediary convolution layers require $170 \mathrm{~ms}$, and proposed regional network layer requires $30 \mathrm{~ms}$, in a $1.7 \mathrm{Ghz}$ TitanX CPU. 
any investment requirement. Its own resources plus access to credit place it far ahead of its competitors, regarding access to funds for investment.

Apple's repeatedly successful strategy of consolidating its brand and market presence over time could allow it to continue to replicate its prior successes with new products [50], possibly including AV.

While Apple does utilize relatively well-known technologies, such as LIDAR, it has access to a software developed in-house, VoxelNet, which could represent a significant leap in 3D imaging and recognition, which could lead to an especially important technological competitive advantage in relation to its competitors.

A common opportunity to all companies in the AV market is that the Paris Agreement could stimulate AV sales.

Another common opportunity to all companies in the AV market lies in adopting the use of sodium batteries (or another technology), as opposed to lithium batteries, since sodium is abundant and would not represent a limiting supply factor [29].

\subsubsection{Threats for Apple}

Tesla, as other players in the AV Market, may face lack of acceptance by drivers who may not adapt well to onboard computers.

If Apple does not increase its real-world data acquisition for its AV platform, it may fall behind other competitors that currently gather more AI data.

As is the case with other players in the AV market, Waymo may face battery issues relating to lithium scarcity, prices, and technology.
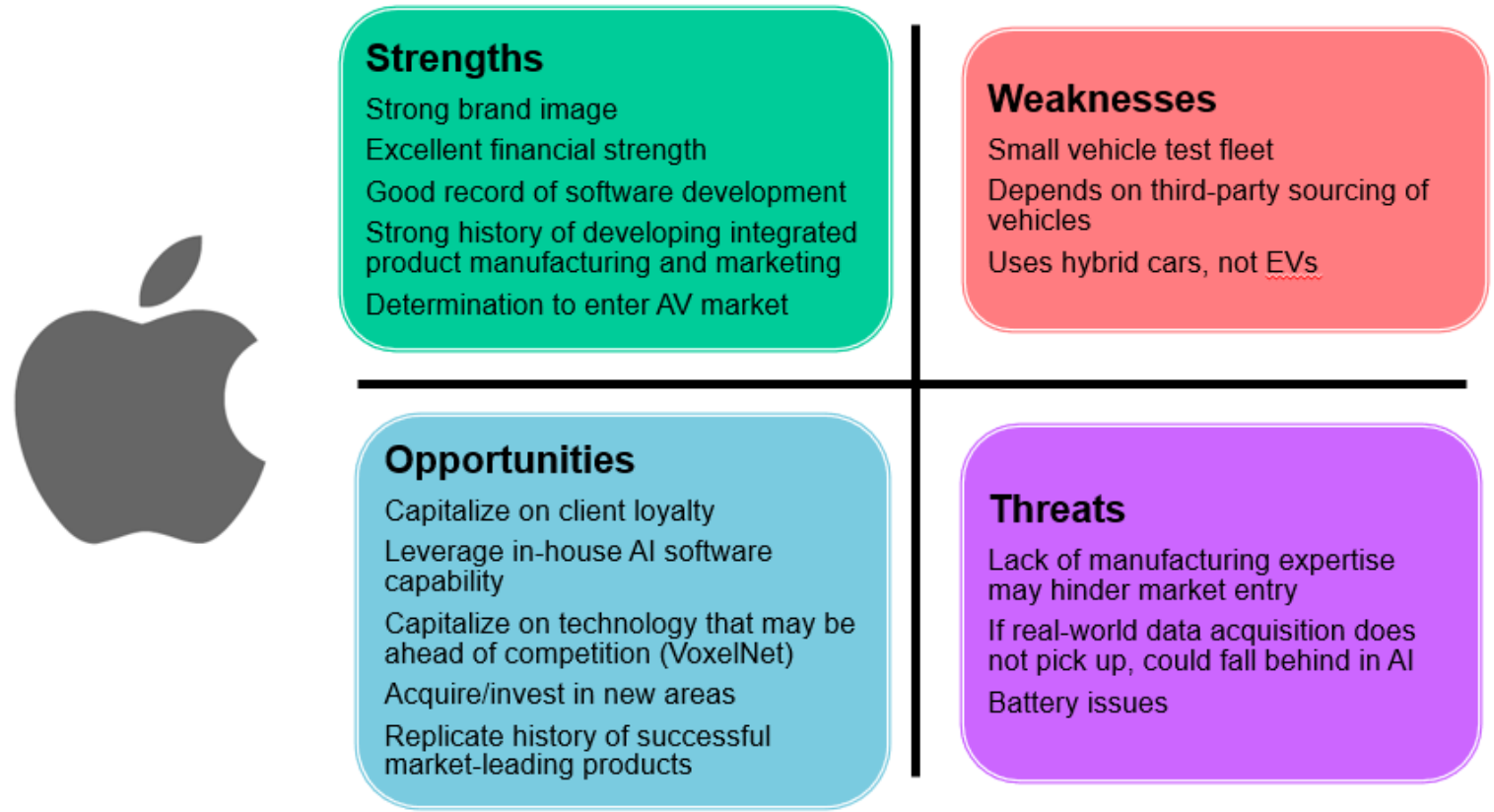

Figure 4. SWOT matrix for Apple.

\section{Relative importance of factors for market dominance}

Five factors were considered to be key to market dominance among AV companies: Current market presence, Brand Strength, Technology, Financial Health and Marketing Strategy.

Current market presence was chosen as a factor since an established leadership position imposes at least initial barriers to overcome it. Brand strength was chosen as a factor since it expresses the market identity of each company, responsible for how clients are connected to their products. Technology was chosen as a factor due to its importance in the development of a desirable product. This includes the ability of the AI system to perform without internet reliance, resulting in a resilient architecture of the 3D recognition system. Financial Health was chosen as a factor because it can allow or impede investment 
plans regarding product development. Marketing strategy was chosen as a factor because it can define the eventual success of a product in the market.

In the short-term, market dominance is deemed to be most closely related to current market presence, since it would take some time for any player to gain dominance over the current market leader, even if underlying conditions favor a change in leadership position. In the medium-term, market dominance is deemed to be most closely associated with how well a company can become a relevant supplier to other players, allowing even conventional auto makers to immediately participate in the AV market by utilizing its packages of key AV elements, such as AI integrated circuit topology, or other AV software solution packages. In the long-run, market dominance is deemed to be related to how well a company can integrate all elements of AV into a proprietary system.

\section{Market dominance assessment}

Currently, Tesla is the best positioned company in the AV market, among the three studied, since it is the only one in the study group that currently produces AV and has an effective presence in the market. Tesla also leads in miles of AV data collected, and has a large big data. Thus, Tesla is the AV market leader, and may remain so for some time (short-term). However, in the medium-term, say within five years, Tesla may lose market share to its competitors, since its financial position may prevent it from making necessary large-scale investments.

Waymo has experience in developing and marketing software solutions, and it demonstrated that it can create intelligent systems for AV. It also has a faster and more efficient means of AI learning to support AV platforms. Thus, despite not demonstrating an interest in producing its own vehicles, it could sell or license its software technology (AV software solution packages) to other vehicle manufacturers, just as Alphabet, Waymo's parent company, has become the software giant it is by marketing software solutions to other companies. Waymo may indeed be considering this strategy, since it continues to conduct tests on vehicles from other automotive manufacturers. Thus, Waymo could possibly surpass Tesla in the medium-term, say after five years, by becoming a preferred source of AV software solution packages for vehicle manufacturers.

Given Apple's stated strategic objective of market dominance, Apple will likely continue to focus on developing its own integrated (hardware and software) AV solution as a top-quality product, since that is the history of its other, tremendously successful products, such as its smartphones, that it developed on its own and then brought to market. This focus could allow Apple to satisfy clients that are loyal to its brand, and allow it to compete in the AV market with a superior product. Apple may already be building an insurmountable quality and technological advantage with which to reach market dominance, with its VoxelNet software (largely internet-independent), and its device interconnection software.

Apple's experience has shown that this process may take a relatively long time to reach market dominance. However, over a longer-term horizon of, say, twenty years, Apple's ability to integrate all elements of AV into a proprietary system, its unique AV technologies, its marketing strategy, and its financial resources could lead to Apple eventually surpassing Waymo as the ultimate AV market leader.

\section{Results}

Given the most important strengths, weaknesses, opportunities and threats of each of the three companies studied, the relative dominance factors that act as drivers for market leadership, and the time frames in which these factors may operate, we propose a graph (Figure 5) that represents the probable relative leadership position of each of the three companies studied in the EV market, over a relative time scale from short to medium to long-term, which we suggest may approximately correspond to five, ten and twenty years, respectively. 
This analysis indicates that the most significant drivers for short-term market dominance suggest that Tesla will retain market leadership, over the short-term. The increasing value of software solutions developed by Waymo will give it the lead, later on. However, Apple's track record in developing market-dominant products, suggests that a similar performance could characterize Apple's entry into the AV market. Thus, the most significant drivers for long-term market dominance, as well as its technological advantages, suggest that Apple will be the eventual AV market leader.

\section{Market \\ share}

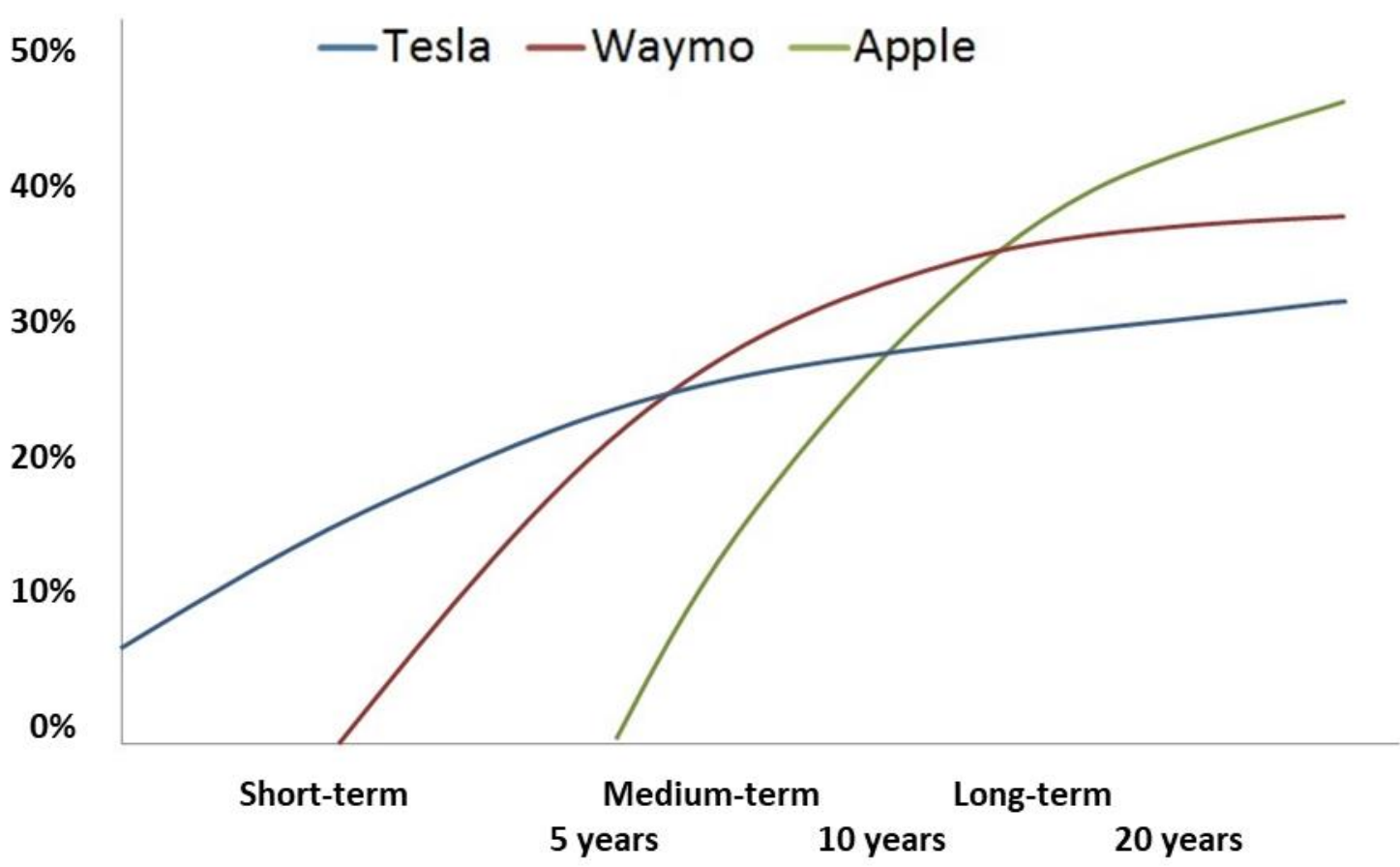

Figure 5. Proposed relative market dominance by the three companies studied, over an approximate relative time scale extending to twenty years.

\section{Discussion}

The relative dominance scenarios by each company are subject to changes, if unforeseen technological breakthroughs are achieved by any one of them, regarding capabilities in handling big data or AI applications, or if their current relative financial health situation changes significantly.

Furthermore, many countries still do not have adequate legislation to allow full development of the capabilities and benefits of the AV market.

However, having a comprehension of which players in the AV market will likely exhibit market dominance, and why, is very valuable to all market players, as they develop their own strategies. Recognizing the strengths, weaknesses, opportunities and threats pertaining to the various AV players and their corporate strategies may allow preemptive policies to be adopted, which could leverage strengths and opportunities, and mitigate weaknesses and threats.

Furthermore, the trends observed and the results of this study provide information that legislators should consider, when planning legislation to ensure that the industry and consumers are best served.

Finally, because the AV market is a worldwide phenomenon, the findings and results from this study may provide useful insights, as countries seek to strengthen their own AV industries as part of their national policies. This possibility suggests the need for further 
analyses of the geopolitical implications of such national strategies, based on the results obtained herein.

\section{References}

1. Litman, T. Autonomous vehicle implementation predictions. Victoria Transport Policy Institute, v. 28 , 2013. http://leempo.com/wp-content/ [12 Oct 2020].

2. Allied Market Research. Autonomous Vehicle Market Outlook - 2026. Online May 2018. Available at https://www.alliedmarketresearch.com/autonomous-vehicle-market [12 Oct 2020].

3. Dillet, R. Tim Cook says Apple's car project is 'the mother of all AI projects'. TechCrunch, online 17 Jun 2017. Available at https://techcrunch.com/2017/06/13/tim-cook-says-apples-car-project-is-the-mother-of-all-ai-projects/ [12 Oct 2020].

4. Waymo. Technology. 2017. Available at https://waymo.com/tech [12 Oct 2020].

5. USA Today. Tesla announces fully self-driving cars. Online 19 Oct 2016. Available at https://www.usatoday.com/story/tech/news/2016/10/19/tesla-announces-fully-self-driving-fleet/92430638/.

6. Gurel, E.; Tat, M. SWOT analysis: a theoretical review. The Journal of International Social Research, v. 10. N. 51, Aug 2017, doi: 10.17719/jisr.2017.1832.

7. Diakakia, C., Papageorgiou, M.; Papamichail, I.; Nikolos, I. Overview and Analysis of Vehicle Automation and Communication Systems from a Motorway Traffic Management Perspective. in Transportation Research Part A Policy and Practice. April 2015, doi: 10.1016/j.tra.2015.03.015.

8. Cavazza, B.H.; Gandia, R.M.; Antonialli, F.; Zambalde, A.L.; Nicolaï, I.; Sugano, J.Y.; Neto, A. de M. Management and business of autonomous vehicles: a systematic integrative bibliographic review. Int. J. Automotive Technology and Management, Vol. 19, N. 1/2, 2019, doi 10.1504/IJATM.2019.098509.

9. Stobbe, J.J.Jr. A Strategic Audit of Tesla. Honors Theses, University of Nebraska-Lincoln. 142. Available at https://digitalcommons.unl.edu/honorstheses/142 [26 Apr 2020].

10. Garrison, W.L.; Levinson, D.M. The Transportation Experience. Oxford University Press. 2nd Ed. 2014.

11. Popkova, E.G.; Ragulina, Y.V.; Bogoviz, A.V. Industry 4.0: Industrial Revolution of the 21st Century. Springer. 2018. ISSN-21984190 .

12. Ozguner, U.; Acarman, T.; Redmill, K. Autonomous Ground Vehicles. Artech House. 2011. ISBN-13: 978-1-60807-192-0.

13. Tian, Y.; Pei, K.; Jana, S.; Ray, B. DeepTest: Automated testing of deep-neural-network-driven autonomous cars. Proceedings of the 40th International Conference on Software Engineering, ICSE 2018, Gothemburg. Doi 10.1145/3180155.3180220.

14. Shuttleworth, J. SAE J3016 - Levels of Driving Automation. SAE International, online 7 Jan 2019. Available at https://www.sae.org/news/2019/01/sae-updates-j3016-automated-driving-graphic [12 Oct 2020].

15. Germany Trade \& Invest. Electromobility in Germany: Vision 2020 and Beyond. Issue 2015/2016. Available at https://www.gtai.de/GTAI/Content/EN/Invest/_SharedDocs/Downloads/GTAI/Brochures/Industries/electromobility-in-germany-vision-2020-and-beyond-en.pdf? $=3$ [12 Oct 2020].

16. KPMG. Autonomous Vehicles Readiness Index: Assessing countries' openness and preparedness for autonomous vehicles, 2018. Available at https://assets.kpmg/content/dam/kpmg/nl/pdf/2018/sector/automotive/autonomous-vehicles-readiness-index.pdf [12 Oct 2020].

17. Villasenor, J. Products Liability and Driverless Cars: Issues and Guiding Principles for Legislation. Brookings Institute, Washington. Online 24 Apr 2014. Available at https://www.brookings.edu/research/products-liability-and-driverless-cars-issuesand-guiding-principles-for-legislation/ [12 Oct 2020].

18. Tesla. Tesla's mission is to accelerate the world's transition to sustainable energy. Corporate site. Online 2018a. Available at https://www.tesla.com/about [12 Oct 2020].0

19. Ross, P.E. Tesla Reveals Its Crowdsourced Autopilot Data. IEEE-Spectrum, online 26 May 2016. Available at https://spectrum.ieee.org/cars-that-think/transportation/self-driving/tesla-reveals-its-crowdsourced-autopilot-data [12 Oct 2020].

20. Golson, J. Tesla's new Autopilot will run in 'shadow mode' to prove that it's safer than human driving. The Verge, online 19 Oct 2016. Available at https://www.theverge.com/2016/10/19/13341194/tesla-autopilot-shadow-mode-autonomous-regulations [12 Oct 2020].

21. Tesla. Model 3. Corporate site. Online 2018b. Available at https://www.tesla.com/model3 [12 Oct 2020].

22. U.S. S.E.C. (SECURITIES AND EXCHANGE COMMISSION). Form 10-k - Tesla, Inc. Washington, D.C. 2018. Available at https://www.sec.gov/Archives/edgar/data/1318605/000156459018002956/tsla-10k_20171231.htm [12 Oct 2020].

23. O'Kane, S. How Tesla and Waymo are Tackling a Major Problem for Self-driving cars: Data. Mobility21. The Verge, online 19 Apr 2018c. Available at https://mobility21.cmu.edu/how-tesla-and-waymo-are-tackling-a-major-problem-for-self-driving-carsdata/ [12 Oct 2020].

24. Eliot, L. LIDAR Put Into A Tesla-And The World Doesn't Come To An End. Forbes, online 8 Oct 2019. Available at https://www.forbes.com/sites/lanceeliot/2019/10/08/lidar-put-into-a-teslaand-the-world-doesnt-come-to-an-end/\#720ba4437f87 [12 Oct 2020].

25. Hull, D.; Recht, H. Tesla Doesn't Burn Fuel It Burns Cash. Bloomberg, online 30 Apr 2018. Available at https://www.bloomberg.com/graphics/2018-tesla-burns-cash/ [26 Apr 2020].

26. Isidore, C. Tesla has a problem. Maybe a big Problem. CNN Business, online 28 Mar 2018. Available at https://money.cnn.com/2018/03/28/news/companies/tesla-model-3-cash-crunch/index.html [12 Oct 2020]. 
27. The Verge. Tesla's record 2019 has bought it some breathing room. Online 29 Jan 2020. Available at https://www.theverge.com/2020/1/29/21113987/tesla-q4-2019-earnings-results-profit-revenue-model-3 [20 Dec 2020].

28. CNBC. Tesla shares jump 5\% after reporting fifth consecutive quarter of profits. Online 21 Oct 2020 . Available at https://www.cnbc.com/2020/10/21/tesla-tsla-earnings-q3-2020.html [20 Dec 2020].

29. Evarts, E.C. Reports: Tesla working to develop its own batteries. Green Car Reports, online 28 Jun 2019. Available at: https://www.greencarreports.com/news/1123834_reports-tesla-working-to-develop-its-own-batteries [12 Oct 2020].

30. Hawkins, A J. Elon Musk still doesn't think LIDAR is necessary for fully driverless cars. The Verge, online 7 Feb 2018. Available at https://www.theverge.com/2018/2/7/16988628/elon-musk-lidar-self-driving-car-tesla [26 Apr 2020].

31. Medium. Waymo reaches 5 million self-driven miles. Online 28 Feb 2018. Available at https://medium.com/waymo/waymoreaches-5-million-self-driven-miles-61fba590fafe [12 Oct 2020].

32. Ross, P.E. Waymo Shows Off Its Home-Grown Robocar Hardware. IEEE-Spectrum, online 9 Jan 2017. Available at https://spectrum.ieee.org/cars-that-think/transportation/self-driving/waymo-shows-off-its-doityourself-robocar-hardware [12 Oct 2020].

33. Alphabet. Alphabet Announces Fourth Quarter and Fiscal Year 2019 Results. Online 3 Feb 2020. Available at https://abc.xyz/investor/static/pdf/2019Q4_alphabet_earnings_release.pdf [20 Dec 2020].

34. O'Kane, S. Waymo strikes a deal to buy 'thousands' more self-driving minivans from Fiat Chrysler. The Verge, online 30 Jan 2018a. Available at https://www.theverge.com/2018/1/30/16948356/waymo-google-fiat-chrysler-pacfica-minivan-self-driving [12 Oct 2020].

35. Sage, A. Waymo unveils self-driving taxi service in Arizona for paying customers. Reuters, online 5 Dec 2018 . Available at https://www.reuters.com/article/us-waymo-selfdriving-focus/waymo-unveils-self-driving-taxi-service-in-arizona-for-payingcustomers-idUSKBN1O41M2 [12 Oct 2020].

36. Carlton, J. Apple: The inside Story of Intrigue, Egomania, and Business Blunders. 1st ed. New York: Random House Inc., 1997. ISBN-13: 978-0812928518. [26 Apr 2020].

37. Yoffie, D.; Kim, R. Apple Inc. in 2010. HBS Case No. 710-467, 2010. Harvard Business School Strategy Unit. Available at https://ssrn.com/abstract=1607809 [12 Oct 2020].

38. The Brand Specialist. Apple's 'Creative' Brand Position. Online 10 Nov 2018. Available at https://www.thebrandspecialist.com/apples-creative-brand-position/ [12 Oct 2020].

39. Apple. Privacy. Corporate site. Online 2018. Available at https://www.apple.com/privacy [12 Oct 2020].

40. Apple. Apple Reports Fourth Quarter Results. Corporate site. Online 29 Oct 2020. Available at https://www.apple.com/newsroom/2020/10/apple-reports-fourth-quarter-results/ [20 Dec 2020].

41. Davies, R. Apple becomes world's first trillion-dollar company. The Guardian, online 2 Aug 2018. Available at https://www.theguardian.com/technology/2018/aug/02/apple-becomes-worlds-first-trillion-dollar-company [12 Oct 2020].

42. Rogers, C.; Ramsey, M.; Wakabayashi, D. Apple Hires Auto Industry Veterans. The Wall Street Journal, online 20 Jul 2015. Available at https://www.wsj.com/articles/apple-hires-auto-industry-manufacturing-veteran-1437430826 [12 Oct 2020].

43. Heisler, Y. Apple hires another major talent for its top-secret car project. BGR, online 21 Jul 2015. Available at https://bgr.com/2015/07/21/apple-car-self-driving-rumors/ [12 Oct 2020].

44. Gurman, M.; Hull, D. Apple Hires Tesla Engineer With Interior Design Experience. Bloomberg, online 22 Jul 2019. Available at https://www.bloomberg.com/news/articles/2019-07-22/apple-hires-tesla-engineer-with-interior-design-experience [12 Oct 2020].

45. Huilgol, M. Apple's Latest Patent Will Allow Autonomous Cars to Communicate with Other Vehicles and Pedestrians. iPhone Hacks, online 10 Jun 2019. Available at http://www.iphonehacks.com/2019/06/apple-project-titan-autonomous-patent.html [12 Oct 2020].

46. Zhou, Y.; Tuzel, O. VoxelNet: End-to-End Learning for Point Cloud Based 3D Object Detection. arXiv preprint arXiv:1711.06396v1, online 17 Nov 2017. Available at https://arxiv.org/pdf/1711.06396.pdf [12 Oct 2020].

47. Tapalaga, A. Apple Is Gaining Back Market Share in the Smartphone Industry. Medium.com. Online 11 Mar 2020. Available at https://medium.com/@atapalaga97/apple-is-gaining-back-market-share-in-the-smartphone-industry-64ad5eb59f4a [26 Apr 2020].

48. NICAS, J. Apple, Spurned by Others, Signs Deal With Volkswagen for Driverless Cars. New York Times, online 24 May 2018. Available at https://www.nytimes.com/2018/05/23/technology/apple-bmw-mercedes-volkswagen-driverless-cars.html [12 Oct 2020].

49. O'Kane, S. Apple now has more self-driving test vehicles in California than Waymo. The Verge, online 20 Mar 2018b. Available at https://www.theverge.com/2018/3/20/17143708/apple-self-driving-google-waymo-california-uber [12 Oct 2020].

50. Johnson, K.; Li, Y.; Phan, H.; Singer, J.; Trinh, H. The Innovative Success that is Apple, Inc. Theses, Dissertations and Capstones, Marshall Digital Scholar, Marshall University. Paper 418, 1-1-2012. Available at https://mds.marshall.edu/cgi/viewcontent.cgi?article=1420\&context=etd [12 Oct 2020]. 